\title{
ARP2/3-Mediated Actin Nucleation Associated With Symbiosome Membrane Is Essential for the Development of Symbiosomes in Infected Cells of Medicago truncatula Root Nodules
}

\author{
Aleksandr Gavrin, Veerle Jansen, Sergey Ivanov, Ton Bisseling, and Elena Fedorova \\ Laboratory of Molecular Biology, Wageningen University, Droevendaalsesteeg 1, 6708PB, Wageningen, The Netherlands
}

Submitted 19 December 2015. Accepted 15 January 2015.

\begin{abstract}
The nitrogen-fixing rhizobia in the symbiotic infected cells of root nodules are kept in membrane compartments derived from the host cell plasma membrane, forming what are known as symbiosomes. These are maintained as individual units, with mature symbiosomes having a specific radial position in the host cell cytoplasm. The mechanisms that adapt the host cell architecture to accommodate intracellular bacteria are not clear. The intracellular organization of any cell depends heavily on the actin cytoskeleton. Dynamic rearrangement of the actin cytoskeleton is crucial for cytoplasm organization and intracellular trafficking of vesicles and organelles. A key component of the actin cytoskeleton rearrangement is the ARP2/3 complex, which nucleates new actin filaments and forms branched actin networks. To clarify the role of the ARP2/3 complex in the development of infected cells and symbiosomes, we analyzed the pattern of actin microfilaments and the functional role of ARP3 in Medicago truncatula root nodules. In infected cells, ARP3 protein and actin were spatially associated with maturing symbiosomes. Partial ARP3 silencing causes defects in symbiosome development; in particular, ARP3 silencing disrupts the final differentiation steps in functional maturation into nitrogen-fixing units.
\end{abstract}

Soil bacteria, collectively known as rhizobia, establish symbiotic relationships with the leguminous plants. Within root nodules, the bacteria are hosted in specialized cells in the compartments called symbiosomes. The membrane of symbiosomes is derived from the host cell plasma membrane. The symbiosis between legume and rhizobia represents a striking example of a prolonged intracellular lifestyle of bacteria in plant cells inside specialized membrane compartments. However, the mechanisms by which the architecture of symbiotic cells is modified to accommodate intracellular bacteria are not clear.

Nucleotide sequence data for Medicago ARP3 is available under accession number Medtr8g089630.1.

Current address for A. Gavrin: Plant Molecular Biology Laboratory, School of biological science, The University of Sydney, Australia.

Current address for S. Ivanov: Boyce Thompson Institute for Plant Research, Ithaca, New York, United States.

Corresponding author: E. Fedorova; E-mail: elena.fedorova@wur.nl; Telephone: +31-(0)317-482036; Fax: +31-(0)317-418094

○ 2015 The American Phytopathological Society
The intracellular architecture of plant cells relies heavily on the cytoskeleton and, particularly, on the actin cytoskeleton. Actin microfilament rearrangement is linked to the positioning of organelles and influences cell shape as well as providing a roadway for the transport of membrane vesicles (Pollard and Cooper 2009; van der Honing et al. 2007; Yanagisawa et al. 2013). The change of actin patterns is largely dependent on actin filament assembly and growth, which is, in turn, a function of actin nucleation and polymerization (Avisar et al. 2009; Sinclair et al. 2009). Actin monomers (globular G-actin) are polymerized into filaments with a characteristic architecture and polarity. The rate-limiting step for polymerization is the creation of trimeric actin seeds, a process known as nucleation (Pollard 2007). Two complexes regulate this process, proteins of the formin group and the actin-related proteins 2 and 3 (ARP2/ 3 ) complex. The formation of straight filaments de novo depends on formin (Aspenström 2010; Deeks et al. 2002; Dominguez 2009). The ARP $2 / 3$ complex creates nucleation points on the sides of existing actin filaments, leading to branched networks (Pollard 2007). The ARP2/3 complex consists of seven subunits, two actin-related proteins, ARP2 and ARP3, stabilized in an inactive state by five other subunits (ARPC1, ARPC2, ARPC3, ARPC4, and ARPC5). The complex has numerous nucleation promoting factors (NPF), which have been intensively studied in animals and yeasts (Campellone and Welch 2010; Pollard and Cooper 2009; Rotty et al. 2013). For enteropathogenic bacteria in animal cells, a common strategy for the establishment of an intracellular lifestyle is the manipulation of host cell actin via the ARP2/3 actin nucleating complex (Welch and Way 2013).

In plants, the homologs of ARP3 detected by heterologous antibodies in tobacco and maize were localized along cortical actin filaments and to multivesicular bodies (Van Gestel et al. 2003). The activation of ARP $2 / 3$ in most plant species is dependent on the SCAR/WAVE (suppressor of cAMP receptors/ WASP family verprolin homologous) family of NPF (Yanagisawa et al. 2013).

The role of actin and ARP2/3 in rhizobium-legume symbiosis has been mainly studied at the early stages of the interaction. This showed, for example, that Nod factors, signal molecules from rhizobia, cause the deformation and the fragmentation of a fine mesh of actin in root hairs (Blancaflor et al. 2006; Cárdenas et al. 1998; de Ruijter et al. 1999; Timmers 2008). The importance of the ARP $2 / 3$ pathway for symbiosis was shown recently (Yokota et al. 2009; Miyahara et al. 2010; Hossain et al. 2012). For example, the $M$. truncatula ortholog of $N A P 1$, a component of the SCAR/WAVE complex, is operational for infection thread growth in the early stages of symbiosis 
(Miyahara et al. 2010). Exposure of root hairs of Phaseolus vulgaris to Rhizobium etli Nod factors resulted in a rapid increase in the number of filamentous actin (F-actin) newly available plus ends, which probably represent sites of polymerization, and in the relocalization of F-actin plus ends to infection thread initiation sites (Zepeda et al. 2014)

However, the mechanisms of actin reformation in the intracellular stage of symbiosis-when the rhizobia are becoming nitrogen-fixing organelles-were not examined, despite the documented changes in microfilament organization within the infected cells of root nodules (Davidson and Newcomb 2001; Fedorova et al. 2007; Whitehead et al. 1998).

The aim of this work was to study the role of actin nucleating factor ARP3 in the intracellular accommodation and development of symbiosomes in M. truncatula nodules. We found that, during the transition from an immature stage of development to the stage of nitrogen fixation, the actin pattern in an infected cell undergoes prominent reorganization. The actin microfilaments in the cytoplasm of the host cell form a network around the mature symbiosomes. Silencing of ARP3 negatively affects the symbiosome maturation.

\section{RESULTS}

\section{The actin configuration changes during intracellular accommodation of rhizobia.}

To determine the role of actin in host cell development, we first visualized F-actin in root nodules using phalloidin with a fluorescent tag. Medicago nodules (14 and 21 days postinoculation [dpi]) were fixed and were hand-sectioned along the longitudinal axis. These sections were incubated with phalloidin (discussed below) and were subsequently analyzed by confocal microscopy. The nodule meristem displays a typical root meristem cell actin configuration; in interphase cells, there is a fine F-actin network in the cortex and a radial array of actin cables tethering the nucleus (Fig. 1A). In differentiated uninfected cells, prominent thick actin cables were located in cytoplasmic strands across the vacuoles, forming a network around the nucleus (Fig. 1B). These transvacuolar strands interconnect the perinuclear and cortical cytoplasm (Ketelaar et al. 2010). This F-actin organization is very similar to that of other differentiated plant cells with an isodiametric type of growth. In new infected cells in which rhizobia colonization is just starting, the actin microfilaments were associated with infection threads but not with freshly released or dividing bacteria (Fig. 1C and D). However at the transition from colonization to functional maturation, the actin microfilament pattern in infected cells changes markedly (Fig. 1D). In later stages of symbiosis in the cells containing growing symbiosomes, we observed the formation of a net of fine actin microfilaments around symbiosomes (Fig. 1E and F). The final step of functional symbiosome differentiation coincides with a radial alignment of symbiosomes in the host cell cytoplasm. The mechanisms that may be controlling this change are discussed below.

\section{ARP3 is localized in the cytoplasm surrounding the symbiosomes.}

To determine the role of the actin pattern change that causes the formation of an actin net around the symbiosomes, we first needed information about the underlying mechanism that might affect the actin network. To study whether the ARP2/3 pathway is involved in the formation of the fine F-actin surrounding symbiosomes, we focused on the ARP3 subunit. Members of genus Medicago have a single ARP3 gene (Medtr8g089630.1). Its expression was studied by reverse transcription-quantitative polymerase chain reaction (qPCR) in roots and nodules 7,14 , and 21 dpi. The expression level of ARP3 is higher in mature 21dpi nodules in comparison with primordia and young 7-dpi nodules. This points to a role in later stages of symbiosis (Fig. 2A). To gain insight into the expression of $A R P 3$ in developmental zones of the nodule, we have used the INRA Symbimix database (Fig. 2B). Symbimix is based on laser-capture microdissection, which was coupled to RNA sequencing and gives the transcriptome of both bacterial and plant partners in five developmental zones of the nodules of M. truncatula (Roux et al. 2014). The analysis of ARP3 expression (Fig. 2B) shows that mature infected cells have the highest level of $A R P 3$ expression versus the meristem and the distal infection zone that contains freshly infected cells (Fig. 2B). However in the data obtained by Limpens et al. (2013) by a similar method, the expression of $A R P 3$ was found to be highest in the zone of infection.

To study the subcellular localization of ARP3 in infected cells, we constructed N-terminal fusion of ARP3 with green fluorescent protein (GFP) under the leghemoglobin (Lb) promoter, which is active only in infected cells. GFP-ARP3expressing nodules were analyzed by confocal microscopy (Fig. 1G). The analysis of subcellular localization of GFPtagged ARP3 in mature infected cells of the fixation zone has shown that ARP3 localized in the host cell cytoplasm; the fluorescence signal of GFP was surrounding the symbiosomes and delineating the plasma membrane (Fig. 1G). Due to the activity of Lb promoter, the GFP labeling was observed in infected cells but was absent in uninfected neighboring cells. In a separate experiment to verify the labeling pattern, we used a specific anti-ARP3 antibody developed for Arabidopsis thaliana (Kotchoni et al. 2009). ARP3 proteins of A. thaliana and $M$. truncatula share over $87 \%$ identity at the level of amino acid sequences. The anti-ARP3 antibodies were tested on Medicago nodule extracts by Western blot analysis. A band of expected size was detected, which shows that anti-ARP3specific antibody may be used in the analysis of Medicago nodules (Fig. 2C). Immunolabeling with specific anti-ARP3 antibody has shown a pattern similar to that observed for GFPtagged ARP3 (Fig. 1H and I).

To study the localization of ARP3 in relation to actin in high resolution, we used electron microscopy immunogold labeling in mature symbiotic cells of nodules expressing GFP-ARP3. For immunolabeling of actin, we used antiactin antibody developed in mouse, followed by a secondary antimouse antibody coupled to 10-nm colloidal gold particles. For immunolocalization of ARP3, we used, in separate experiments, either anti-GFP antibody developed in rabbit or specific anti-ARP3 antibody, which was also developed in rabbit. The secondary antirabbit antibody coupled with 15-nm gold particles were used to reveal the signal (Fig. 3). The signals for actin and for ARP3 were present in the cytoplasm around the symbiosomes in stage 3 according to the developmental stage classification of Vasse et al. (1990) (Fig. 3A and B). These symbiosomes already lost the ability to divide and were growing rapidly. Actin and ARP3 were also associated with small vacuoles (Fig. $3 \mathrm{C}$ and D). The actin network around symbiosomes were structurally similar to the dynamic "actin scaffold" observed around the membrane of the phagosomes by Liebl and Griffiths (2009).

\section{The actin network around symbiosomes is not formed in $\operatorname{dnf} 1$ mutant nodules.}

To clarify the link between the developmental stage of the symbiosomes and the ARP3 pattern, we used Medicago mutant dnfl (Wang et al. 2010). DNF1 gene encodes a symbiosisspecific subunit of the signal-peptidase complex. The mutation of this gene blocks the development of the symbiosomes so they are not able to reach maturity (Van de Velde et al. 2010; Wang et al. 2010). The nodules of the $d n f l$ mutant are unable to 
fix nitrogen, and their infected cells are filled with small symbiosomes at developmental stages 1 and 2 (Fig. 4A and B). Immature symbiosomes may persist, in this stage, up to 10 consecutive cell layers till the senescence starts.

We performed the actin labeling (Fig. 4C) and the immunolocalization of ARP3 using the specific anti-ARP3 antibody on nodule sections of $d n f 1$ mutant (Fig. 4D). The actin pattern in the uninfected cells was similar to what we previously observed (Fig. 1A to C). Actin microfilaments were crossing the vacuoles and delineating the plasma membrane. However, infected cells of $d n f 1$ nodules do not form the subtle actin net enveloping the symbiosomes, as was observed in control nodules (Fig. 1F). The signal for ARP3 outlined the plasma membrane (Fig. 4D) but did not surround the symbiosomes in the same way as was observed in control nodules (Fig. 1G and $\mathrm{H})$. The pattern of ARP3 in uninfected cells was, however, similar to that in uninfected cells of control nodules. We conclude that ARP3 association with symbiosomes depends on the developmental stage; it starts to be assembled in transition between stages 2 and 3 and forms a dense actin network around mature nitrogen-fixing symbiosomes.

The results of immunolocalization of ARP3 in dnf1 mutant nodules suggest that the ARP2/3 complex may be involved in the formation of the F-actin array around membranes of
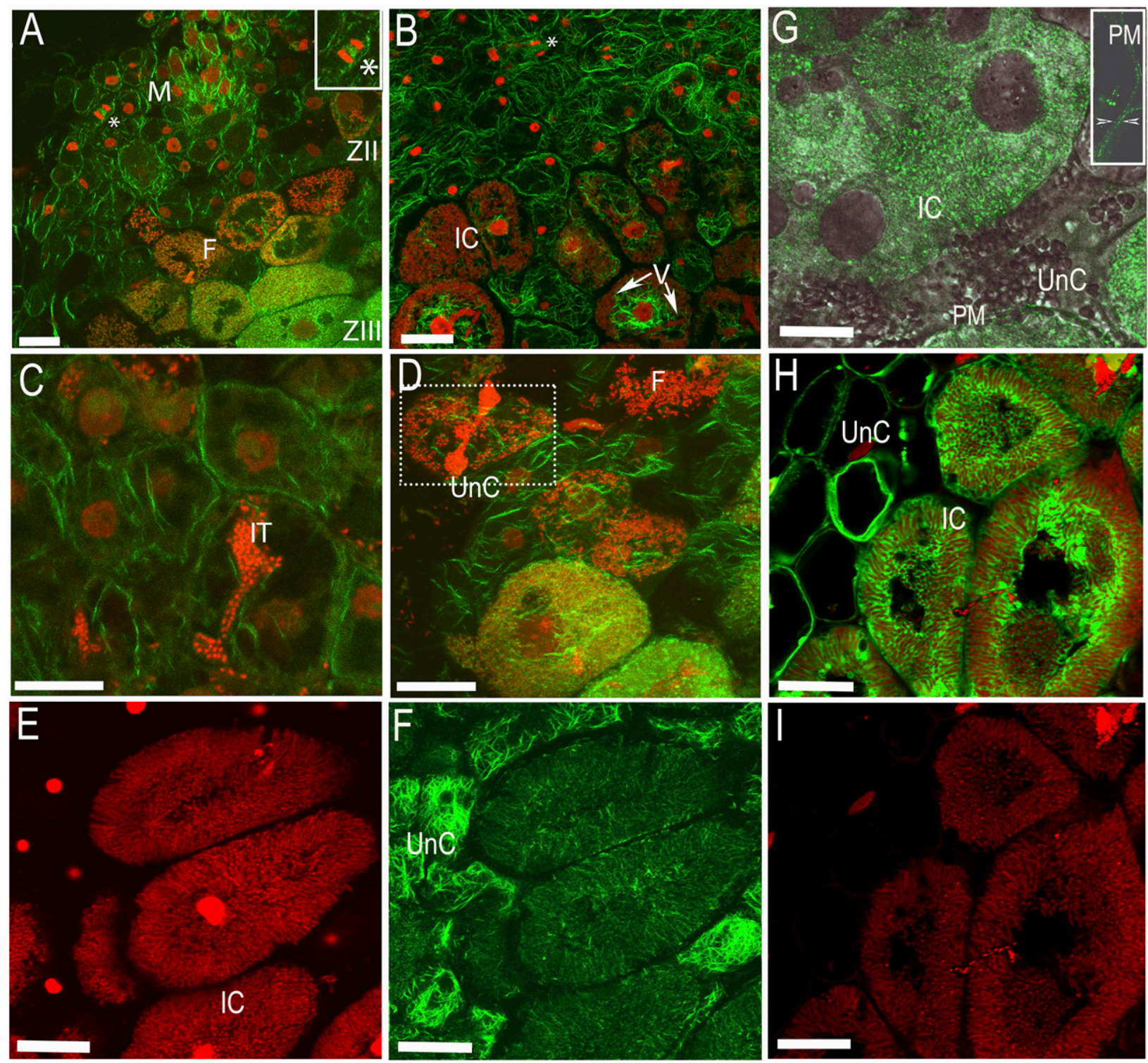

Fig. 1. A to F, Distribution of actin visualized by phalloidin staining (green fluorescence) and $\mathbf{G}$ to $\mathbf{I}$, localization of ARP2/3 in infected cells of Medicago root nodules (green fluorescence). Bacteroids and host cell nuclei were counterstained by propidium iodide (red fluorescence). A, Zonation of the root nodule. The insert shows the dividing cell from the meristem. B, Actin network over the vacuole. Arrows indicate the vacuole. $\mathbf{C}$, Infection thread encompassed by actin filaments. D, Host cell filled with growing stage 2 symbiosomes (Vasse et al. 1990) (outlined by a rectangle). Note that the microfilaments in the host cytoplasm are not yet organized into a net surrounding the symbiosomes and the symbiosomes do not yet have a radial pattern. $\mathbf{E}$ and $\mathbf{F}$, Infected cell housing symbiosomes in stage 3/4, note the radial pattern of symbiosomes and the similar pattern in actin microfilament distribution. To show the actin pattern, the image in F shows only the green channel. G, Localization of ARP3 in infected cells of GFP-ARP3 expressing nodules (noncounterstained differential interference contrast image). The insert displays labeling of plasma membrane (PM) that is visible in the cells in which part of the cytoplasm is not in the focal plane (arrowheads). H, ARP3 immunolocalization using anti-ARP3 antibody, the signal for ARP3 is present in the cytoplasm of mature infected cells around the symbiosomes and in uninfected cells cytoplasm. Note the radial pattern of mature symbiosomes in the infected cell. I, An image displaying only the red channel shows the arrangement of symbiosomes. M, meristem; zII, infection zone; zIII, zone of nitrogen fixation; V, vacuole; IC, infected cell; UnC, uninfected cell; IT, infection thread; F, freshly released bacteria; an asterisk (*)indicates dividing cells. Bars $=20 \mu \mathrm{m}$. 
growing and mature symbiosomes. Therefore, we tested the effect of ARP3 knockdown on the actin configuration and determined its role in symbiosome maturation.

\section{The F-actin network is essential}

for symbiosome development.

To study the role of the ARP2/3 complex in F-actin organization around symbiosomes, we made an RNA interference (RNAi) construct driven by the Lb promoter. Lb promoter becomes active in distal cell layers of the infection zone (Limpens et al. 2009); however, it is heavily up-regulated in mature infected cells of the nitrogen fixation zone. Silencing under the Lb promoter should reduce the level of ARP3 only in infected cells and should not affect uninfected cells. The level of silencing estimated by qPCR, using as a template RNA extracted from pooled nodules from 25 plants, is shown in Figure 2D.

To find out whether the actin configuration is affected due to partial silencing of ARP3, we used phalloidin staining in fresh nodules collected from RNAi-ARP3 transgenic roots; five nodules were analyzed (at $14 \mathrm{dpi}$ ). The configuration of actin filaments in infected cells of RNAi-ARP3 nodules was markedly different from that of control infected cells; actin was present as dots or very short cables (Fig. 4E and F) and the actin net encompassing symbiosomes was absent. The actin microfilament pattern in uninfected cells was not affected (Fig. 4E and F). The intensity of the labeling was similar to control nodules (Fig. 1B and D). We concluded that the partial silencing of ARP3 affects the actin configuration in infected cells.

To investigate the effect of silencing of ARP3 on symbiosome development, we analyzed RNAi-ARP3 nodules by light and electron microscopy (Fig. 5). A total of 25 nodules from two consecutive experiments were analyzed at $15 \mathrm{dpi}$. No defects in the bacteria released from infection threads or host cell structural deviations were detected in the meristem or zone of infection (Fig. 5D). However, around $50 \%$ of the nodules displayed an aberrant phenotype that was observed in distal cell layers, in which maturation of symbiosomes takes place (Fig. $5 \mathrm{E}$ and $\mathrm{F}$ ). In the nodules with an aberrant phenotype, symbiosomes remain markedly smaller throughout the infected zone and they were randomly distributed in the host cytoplasm (Fig. 5E and F). This contrasts with control nodules, in which symbiosomes reached maturity and were radially aligned (Fig. $5 \mathrm{~B}$ and $\mathrm{C}$ ). Vacuole fragmentation also was observed in some of the infected cells in RNAi-ARP3 nodules. In addition to the effect on symbiosome differentiation, in $40 \%$ of the nodules, the plasma membrane was locally detached from the cell wall in infected cells (Fig. 5D, E, and G). The effect on symbiosomes in these cells may, therefore, be indirectly caused by impaired symplast or apoplast transport. However, the defects in symbiosome development were also observed in the nodule cells without visible plasma membrane detachments.

Reduced expression of ARP3 was not lethal for the infected cells, as the plant cells maintained symbiosomes in several
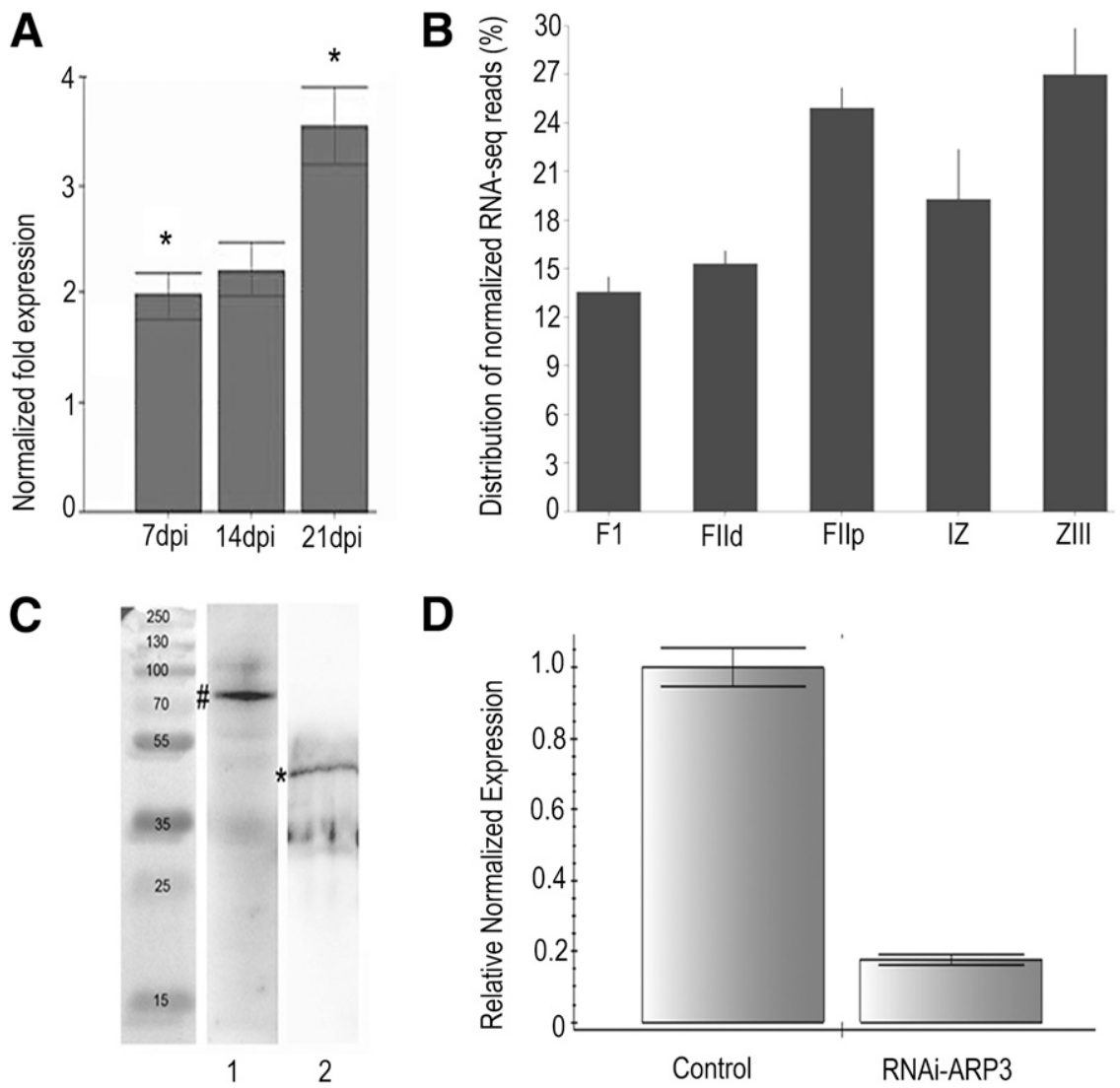

D

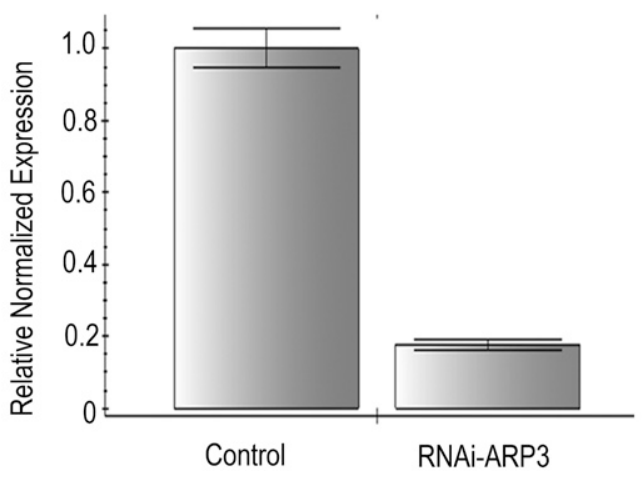

Fig. 2. Expression level of ARP3 and Western blot analysis of nodule extracts. A, ARP3 expression in root nodules at different ages, fold expression relative to uninoculated roots. Error bars represent standard deviation. The difference between the expression of 7-days-postinoculation (dpi) nodules versus 21-dpi nodules is significant $(P<0.05)$. B, Distribution of normalized RNA-seq reads $(\%)$ for Medicago ARP3 (probe Mt0009_10239) in different nodule zones. FI meristematic zone, FIId distal infection zone, FIIp proximal infection zone, IZ interzone II/III, ZIII fixation zone (obtained by using the Symbimix database).C, Western blot analysis of extract from GFP-ARP3 (line 1) and wild-type nodules (line 2) probed with anti-ARP3 antibody shows bands of expected size: The \# symbol indicates the 73-kDa band of green-fluorescent protein-tagged ARP3; the * indicates the 46-kDa band of endogenous ARP3. D, The level of silencing estimated by quantitative polymerase chain reaction analysis of ARP3 expression using a template of RNA extracted from an empty-vector control and RNAiARP3 nodules. The difference between the control and RNAi-ARP3 nodules is significant $(P<0.01)$. Error bars represent standard deviation. 
(approximately 8 to 15 ) cell layers. Ultrastructural analysis of RNAi-ARP3 nodules (Fig. 5G) showed that the host cytoplasm of infected cells contained ribosomes, ER, and mitochondria. However, the symbiosomes remained small and immature and some of them were prematurely lysed.

\section{DISCUSSION}

Actin rearrangement in infected cells in root nodules.

F-actin rearrangement plays a crucial role in mediating the relationships of plant cells in their contacts with biotic agents and pathogens (Day et al. 2011; Henty-Ridilla et al. 2013; Takemoto and Hardham 2004). Cárdenas et al. (1998) and de Ruijter et al. (1999) have shown that Nod factors, the main signaling molecules of symbiosis, affect actin microfilament patterns in root hairs during the establishment of symbiosis. Information about the role of actin in the later stages of symbiosis is scarce; however, some new insights into the molecular mechanism of actin cytoskeletal configuration in infected nodule cells appear. For instance, Marchetti et al. (2013) showed that Medicago microsymbiont Sinorhizobium meliloti cocultured with HeLa cells induced a change of actin pattern. This modification was caused by queuosine, a hypermodifed nucleoside that was produced by bacteria. Although changes of actin pattern have been documented in infected leguminous cells (Davidson and Newcomb 2001; Fedorova et al. 2007; Timmers 2008; Whitehead and Day 1997), the molecular mechanisms of actin microfilament rearrangement in cells containing rhizobia are still unknown.

To address this issue, we investigated the role of actin and ARP2/3-dependent actin nucleation during the intracellular accommodation of rhizobia in root nodule. We have shown that the configuration of actin cytoskeleton changes markedly during the course of symbiosome maturation. Immature dividing symbiosomes in young infected cells of wild-type nodules and in the nodules of $d n f 1$ mutants do not have a net of microfilaments enveloping them. During the phase of symbiosome expansion, actin network around symbiosome membranes may be highly dynamic for supporting vesicle transport and promoting growth. Actin is known to organize plant cytoplasm (Yanagisawa et al. 2013) and may be essential for the organization of narrow zones of cytoplasm around the symbiosome membranes for efficient transport to the membrane.

An actin "scaffold" is gradually assembled around growing symbiosomes and becomes quite prominent around mature nitrogen-fixing units. Analysis of the localization and the functional role of ARP3 in root nodules has shown that ARP3 is operational in the assembly of actin around symbiosomes and is indispensable for the maturation to convert to a nitrogen-fixing units.

\section{How may the ARP2/3 complex contribute to symbiosome maturation?}

In $A$. thaliana, ARP $2 / 3$ is strongly associated with cell membranes of the microsomal fraction from several organelles, including the endoplasmic reticulum (ER), tonoplast, plasma membrane, and the early endosome (Kotchoni et al. 2009; Zhang et al. 2013). It is well-established that symbiosome membrane growth relies on host cell membranes provided in the form of post-Golgi vesicles and ER (Ivanov et al. 2012; Kereszt et al. 2011). The integration of ARP $2 / 3$ into branched actin filament networks may facilitate the physical anchoring of a polymerizing actin filaments on an organelle surface (Yanagisawa
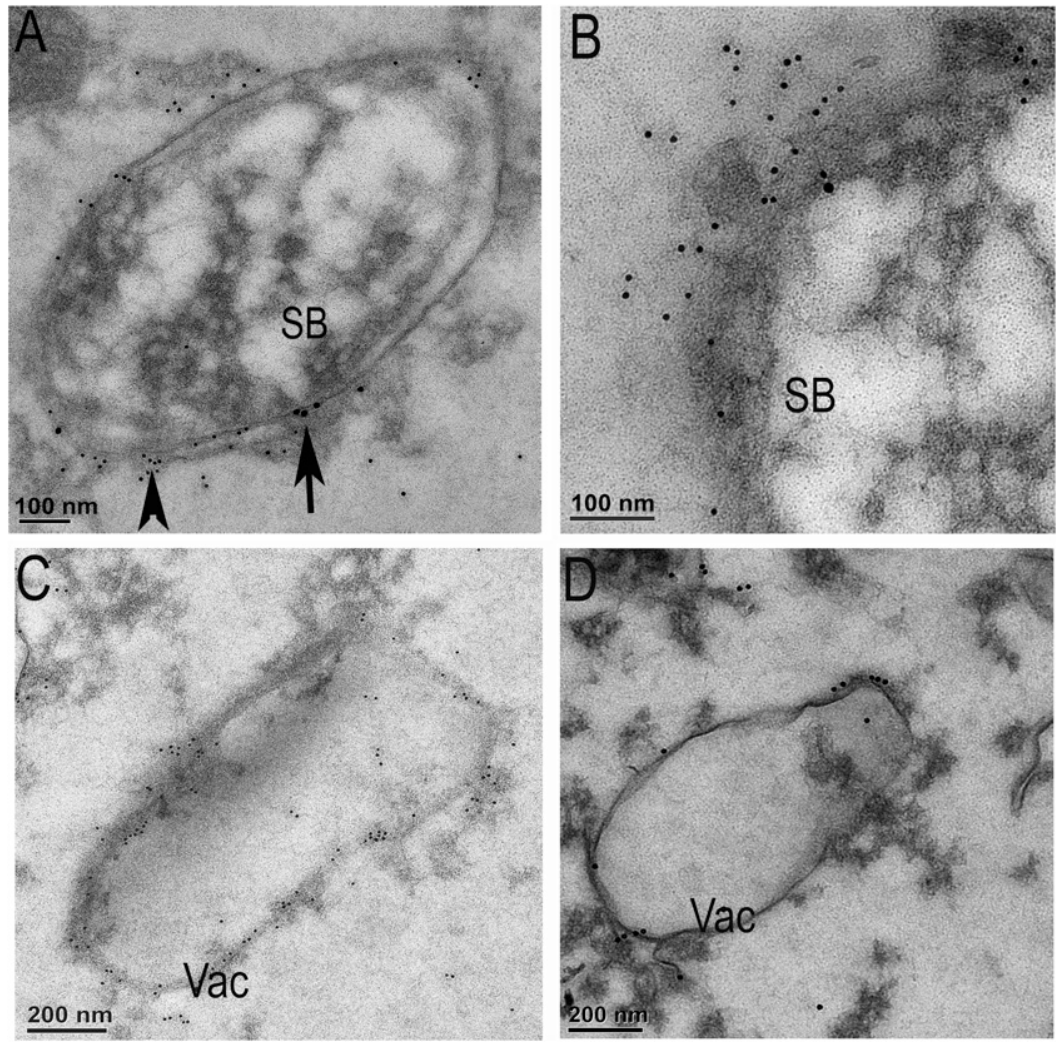

Fig. 3. Immunogold localization of ARP3 and actin in infected cells. A and B, Double labeling. A, Localization of actin (10-nm gold particles) is indicated by the arrowhead, and ARP3 (15-nm gold particles) is indicated by the arrow in infected cells of GFP-ARP3-expressing nodules. The signal is present in the cytoplasm surrounding the symbiosome membrane. B, Microfilaments around the symbiosome membrane labeled for actin (10-nm gold particles). C, Small vacuole labeled by antiactin antibody. D, Small vacuole labeled by anti-green fluorescent protein (GFP) antibody in GFP-ARP3-expressing nodule tissue. SB, symbiosome; Vac, vacuole. 
et al. 2013). Similar mechanisms may be employed during the construction of symbiosome membranes. It is possible that the phenotype we observed in RNAi-ARP3 nodules may be the consequence of nonoptimal association of ARP3 with the membranes of ER and post-Golgi vesicles causing the defects in their trafficking toward the symbiosomes.

In the last phase of symbiosome development, after they reach maturity and start to fix atmospheric nitrogen, actin is arranged around symbiosome membranes to form a network of microfilaments. The formation of this structure depends on the developmental stage; it is a property of maturing and fully mature symbiosomes. Its assembly, therefore, may be linked to symbiosome membrane properties that are developmental stage dependent.
The change of symbiosome membrane identity coincides with the gradual change in actin arrangement

in the cytoplasm surrounding symbiosomes.

In symbiotic infected cells, the identity of freshly released symbiosomes is similar to plasma membrane but, over the course of maturation, they accept late endosome or tonoplast proteins into their membranes (Limpens et al. 2009). Such relocation may be the consequence of host cell vacuole defunctionalization that causes the retargeting of tonoplast-residing proteins toward the symbiosomes (Gavrin et al. 2014). This change in membrane identity, therefore, coincides with the gradual change in actin arrangement around symbiosomes.

The dense actin network around the mature symbiosomes may contribute to maintenance of them as individual units, for
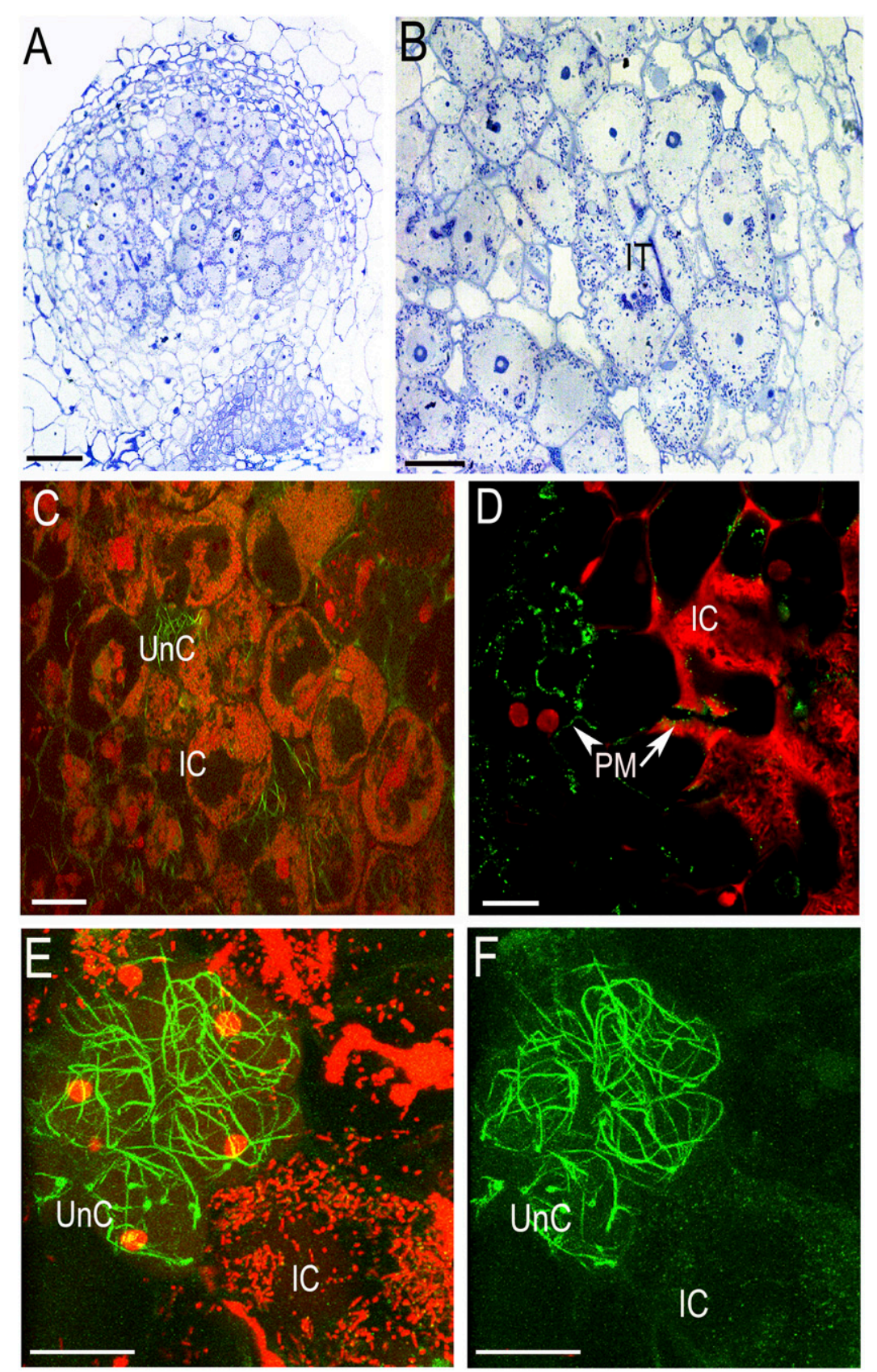

Fig. 4. A to $\mathbf{D}$, Phenotype of $d n f l$ mutant nodules and $\mathbf{E}$ and $\mathbf{F}$, analysis of actin pattern in nodules with partial silencing of ARP3. A and $\mathbf{B}$, light microscopy of the dnf1 nodules. C, Phalloidin staining of actin in the dnf1 nodules. D, The localization of ARP3 in the $d n f 1$ nodules by using anti-ARP3-specific antibody, plasma membrane (PM) is labeled in an infected cell (arrow) and an uninfected cell (arrowhead). E and $\mathbf{F}$, Phalloidin staining of actin in RNAi-ARP3 nodules. The pattern of actin in the infected cell is represented by dots or very short microfilaments. IC, infected cells; UnC, uninfected cells; IT, infection thread. Bars in $\mathrm{A}=50 \mu \mathrm{m}$ and in $\mathrm{B}$ to $\mathrm{F}=20 \mu \mathrm{m}$. 
example, by limiting fusion with the lytic vacuoles as well as preventing homotypic fusion of symbiosomes. The pattern of actin rearrangement around symbiosomes structurally resembles the actin "scaffold" formed around endosomes and phagosomes in animal cells, which prevents fusion with lysosomes (Kumar and Valdivia 2008; Liebl and Griffiths 2009).

In some RNAi-ARP3 nodules, we observed vacuole fragmentation similar to that observed in yeast cells, with mutations of actin and proteins involved in the regulation of nucleation by the ARP2/3 pathway (Eitzen et al. 2002). Vacuole formation defect in the case of actin mutations may be explained by an abnormality of endosome positioning, maturation, and migration toward vacuoles (Gopaldass et al. 2012; Morel et al. 2009; Taunton et al. 2000).

Recently Deeks et al. (2012) and Hawkins et al. (2014) described the plant networked (NET) superfamily of actin-binding proteins. Members of this family couple the actin cytoskeleton with specific membranes, i.e., tonoplast, nuclear membrane, or plasma membrane. It seems worthwhile to test whether the NET protein specific for late endosomes and tonoplasts is retargeted to the symbiosomes at the transition from infection to fixation.

The ARP2/3 complex in plants is essential for some cell types, such as trichomes (Zhang et al. 2005), and may be dispensable in others (Deeks and Hussey 2005). For example, NAP1, a component of the SCAR/WAVE complex that regulates actin polymerization, is operational for appropriate root-hair and infection-thread growth during early stages of infection but dispensable for nodulation (Miyahara et al. 2010). ACTIN-RELATED PROTEIN COMPONENT1 (ARPC1), which constitutes a subunit of ARP2/3, is also essential for rhizobial infection but not for arbuscular mycorrhiza symbiosis in Lotus japonicus (Hossain et al. 2012).

We believe that infected cells of root nodules have specific requirements for $\mathrm{ARP} 2 / 3$, due to the extremely high demand for membrane traffic and the role that the actin network plays in reformation of cytoplasm architecture and symbiosome maturation. We have shown that actin configuration in infected nodule cells changes markedly during symbiosome development. When symbiosomes mature, they become surrounded by actin network. The ARP $2 / 3$ complex is operational in the rearrangement of actin around symbiosomes. We hypothesize that the retargeting of late endosome or tonoplast proteins to the symbiosome membranes allows the recruitment of actin to the symbiosomes and that this recruitment is necessary for their functional maturation.

\section{MATERIALS AND METHODS}

Plant growth conditions, transformation, and inoculation.

Plants of Medicago truncatula Jemalong A17 were grown in the growth chamber with $16 \mathrm{~h}$ of light and temperature of $21^{\circ} \mathrm{C}$. Plants were inoculated with Sinorhizobium meliloti 2011 and were harvested 15 to 21 dpi. Agrobacterium rhizogenes MSU440-based root transformation of $M$. truncatula roots was performed according to Limpens et al. (2004).
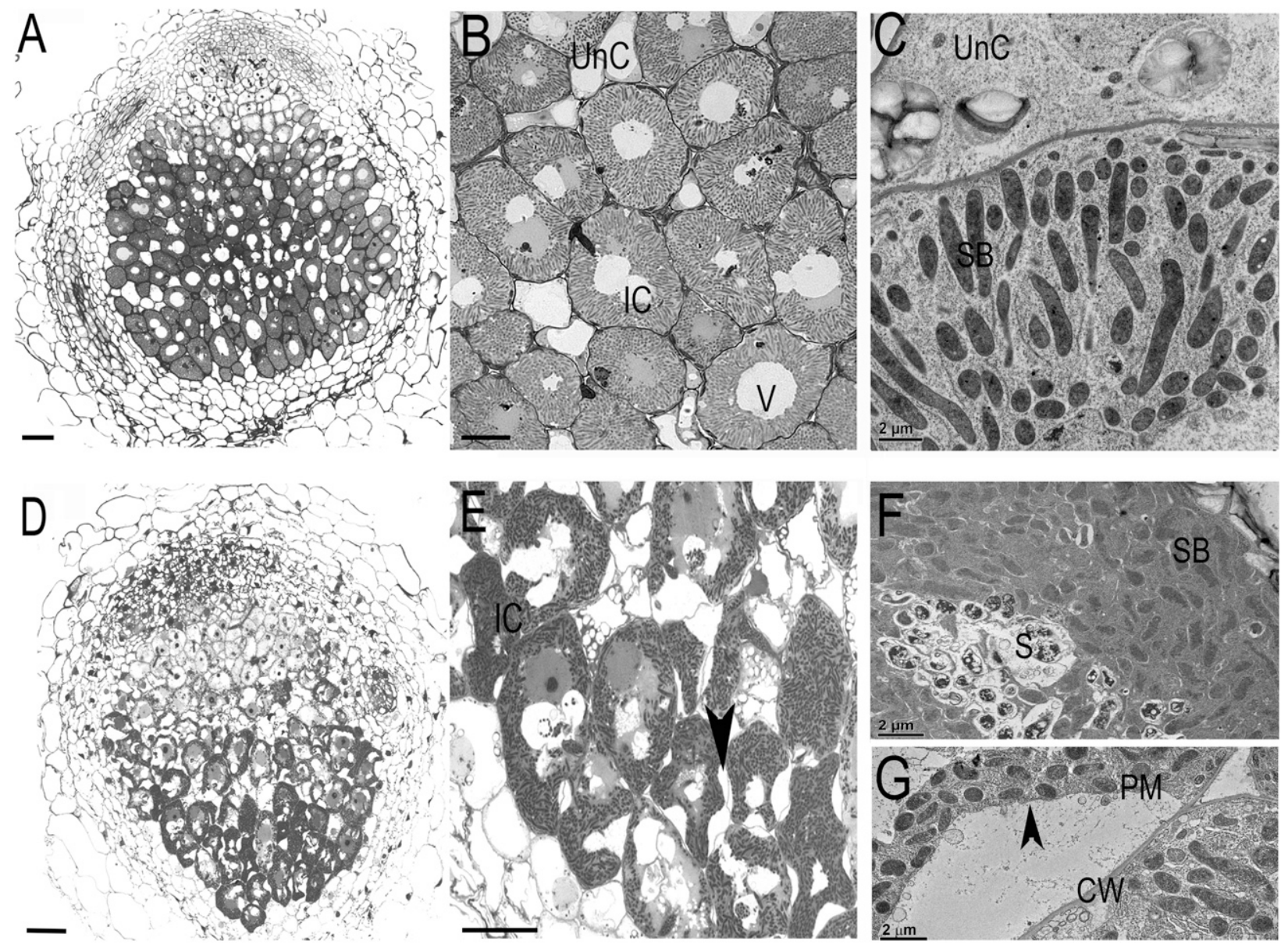

Fig. 5. A, B, D, and E, Light and $\mathbf{C , ~ F , ~ a n d ~} \mathbf{G}$, electron microscopy analyses of RNAi-ARP3 and empty vector control nodules. A to C, Empty vector control nodules and $\mathbf{D}$ to $\mathbf{G}$, RNAi-ARP3 nodules. Silencing did not affect ARP3 expression in the zone of infection; no defects in bacteria release were detected in RNAi-ARP3 nodules in comparison with control (A and D). In the zone of fixation, mature symbiosomes were radially aligned in control nodules (B and C). In RNAi-ARP3 nodules, small undeveloped symbiosomes are freely distributed in the host cytoplasm (E, F, and G). The partial detachment of plasma membrane from the cell wall is indicated by arrowheads (E and G). IC, infected cell; UnC, uninfected cell; It, infection thread; V, vacuole; SB, symbiosome; S, senescent symbiosomes, PM, plasma membrane, $\mathrm{CW}$, cell wall. Bars in $\mathrm{A}$ and $\mathrm{D}=50 \mu \mathrm{m}, \mathrm{B}$ and $\mathrm{E}=25 \mu \mathrm{m}, \mathrm{C}, \mathrm{F}$, and $\mathrm{G}=2 \mu \mathrm{m}$. 
The nodulation assay was performed from two independent experiments. The batch of plants used for transformation contains around 50 plants. The nodules from the transgenic roots of these plants were collected for analysis at 15 and $21 \mathrm{dpi}$. The nodules from at least 10 plants were used for immunolocalization experiments. Fifteen nodules from transgenic roots of different plants were used for analysis of nodule phenotype in the experiments with the silencing of ARP3.

\section{Cloning.}

cDNA for cloning was prepared according to the iScript cDNA synthesis kit protocol (Bio-Rad Laboratories, Inc.) with $1 \mu \mathrm{g}$ of RNA template for cloning and $500 \mathrm{ng}$ of RNA template for qPCR. The desired sequence was amplified by using Phusion High-Fidelity DNA Polymerase (Finnzymes, Finland). Cloning primers: Arp3-cl-F 5'CACCATGGCTCCGGGTATACAAAA3'; Arp3-cl-R 5'TTAATACATCCCC-TTAAAAACAGGA3'. PCR products were purified using a High Pure PCR Products Purification Kit (Roche Diagnostics, Germany).

Entry clone of ARP3gene was generated by TOPO cloning (Invitrogen). The Gateway technology (Invitrogen, USA) was used to create the desired GFP fusion and RNAi destination vectors. pENTR clone of $A R P 3$ was recombined into the following destination vectors using LR Clonase (Invitrogen): pK7WGF2-R (containing LB promoter) creating N-terminal GFP-X fusions and pK7GWIWG2 for RNAi silencing driven by Lb promoter (Limpens et al. 2009).

\section{qPCR analysis.}

Total RNA was extracted from roots and different zones of 14 dpi root nodules using E.Z.N.A. Plant RNA mini kit (Omega Bio-Tek) and transcribed into cDNA using the iScript cDNA synthesis kit (Bio-Rad). qPCR was set up in a $20-\mu l$ reaction system, using iQ SYBR green supermix (Bio-Rad). The expression of ubiquitin was used as an internal control for qPCR analysis. The analysis was performed in three or six replicates. The normalization of results and the creation of the graphs displaying results were performed automatically with Bio-Rad CFX Manager 3.0 software and protocol. Gene-specific primers were designed with Primer-3-Plus software (Untergasser et al. 2007). qPCR primers were Arp3-q-F 5'AGCAATGGAGAGGCGTTAAA3' and Arp3-q-R 5'CGCAGACTGAATGCACTTGT3'.

\section{Sample preparation for transmission electron microscopy.}

Tissue preparation was performed as described earlier (Fedorova et al. 2007). For immunogold analysis with electron microscopy, nodules were fixed in $1 \%$ paraformaldehyde in $50 \mathrm{mM}$ phosphate buffer, were embedded in LR white resin, and were polymerized with UV light at $-20^{\circ} \mathrm{C}$. Thin sections $(60 \mathrm{~nm})$ were cut, using a Leica Reichert Ultracut S microtome; nickel grids with the sections were blocked in 3\% bovine serum albumin (BSA) in phosphate buffered saline (PBS) and were then incubated overnight at $4{ }^{\circ} \mathrm{C}$ with the primary antibody.

Primary antibodies were antiactin developed in mouse (Molecular Probes), dilution 1:20, anti-Arp3 developed in rabbit (generously donated by D. B. Szymanski), dilution 1:50, and anti-GFP developed in rabbit (Molecular Probes), dilution 1:100. The signal was revealed by incubation with the secondary antibodies gold-labeled $(15 \mathrm{~nm})$ goat antirabbit (BioCell), dilution 1:40, or gold-labeled $(10 \mathrm{~nm})$ goat antimouse (BioCell), dilution 1:40, with incubation for $1 \mathrm{~h}$ at room temperature. Sections were contrasted with $2 \%$ aqueous uranyl acetate and lead citrate and were examined using a JEOL JEM 2100 transmission electron microscope.

\section{Confocal laser-scanning microscopy.}

Transgenic roots and nodules were selected using a fluorescent stereomacroscope (Leica MZ FLIII; Leica). Red fluorescent protein DsRED1 was used as a selection marker for the transformed roots and nodules (Limpens et al. 2004). Harvested nodules were manually sectioned in phosphate buffer with $3 \%$ sucrose and were counterstained by propidium iodide $0.01 \%$. Samples were washed with phosphate buffer with sucrose and were transferred to microscope slides. For immunolabeling, nodules were fixed in $1 \%$ paraformaldehyde in $50 \mathrm{mM}$ phosphate buffer, were washed with the same buffer $\left(4^{\circ} \mathrm{C}\right)$, and were manually sectioned. Sections were transferred to the blocking solution (3\% BSA in PBS) and were then incubated overnight at $4^{\circ} \mathrm{C}$ with the primary antibody. Primary antibodies were anti-GFP developed in rabbit (Molecular Probes), dilution 1:250, and anti-Arp3 developed in rabbit (generously donated by D. B. Szymanski), dilution 1:50. Sections were counterstained by propidium iodide $0.01 \%$. Confocal imaging was done on a Zeiss LSM 5 Pascal confocal laser-scanning microscope (Carl Zeiss, GmbH).

\section{Actin labeling with a phalloidin probe.}

Nodule sections were incubated (for $2 \mathrm{~h}$ at room temperature

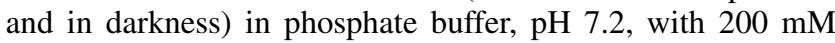
MBS (3-maleimidobenzoic acid N-hydroxysuccinimide ester; Sigma), 0.05\% Triton X-100, and Alexa FluorR 488 phalloidin (Molecular Probes), dilution 1:100. Sections were counterstained and were examined as described earlier.

\section{Western blot analysis.}

The proteins were extracted from root nodules in $0.025 \mathrm{M}$ Tris$\mathrm{HCl}$ buffer containing $1 \mathrm{mM}$ EDTA, $1 \mathrm{mM}$ DTT, and protease inhibitors cocktail (Roche). The probes loaded to the gel measured $15 \mu \mathrm{g}$ per well for extracts from GFP-tagged ARP3 and $45 \mu \mathrm{g}$ per well for extracts from wild-type nodules, to detect the endogenous ARP3. The GFP-tagged isoform was more abundant that the endogenous protein, due to overexpression caused by a strong $\mathrm{Lb}$ promoter used in N-terminal fusion of ARP3 with GFP. The proteins were separated by $12 \%$ sodium dodecyl sulfate-polyacrylamide gel electrophoresis and were blotted to nitrocellulose (Bio-Rad). The membrane was incubated in $3 \%$ BSA, as a blocking agent, followed by primary anti-ARP3specific antibody, 1:50 dilution, followed by secondary antibody, antirabbit immunoglobulin $\mathrm{G}$ peroxidase antibody produced in goat (Sigma), 1:5,000 dilution. The immunosignal was revealed by incubation with Immuno-Star Western chemiluminescent kit (Bio Rad). The blots were photographed by Molecular Imager Chemi Doc XRS+, using Image Lab software in Chemi mode or EpiWhite (for the prestained protein ladder).

\section{ACKNOWLEDGMENTS}

The authors greatly appreciated a generous gift of anti-ARP3 antibody from D. B. Szymanski. We thank T. Ketelaar and H. Franssen for reading and commenting on the manuscript and R. Geurts for fruitful discussions. A. Gavrin was the recipient of a Wageningen University Ph.D. fellowship. E. Fedorova and T. Bisseling are supported by the European Research Council (ERC-2011-AdG294790).

\section{LITERATURE CITED}

Aspenström, P. 2010. Formin-binding proteins: Modulators of formindependent actin polymerization. BBA-Mol. Cell Res. 1803:174-182.

Avisar, D., Abu-Abied, M., Belausov, E., Sadot, E., Hawes, C., and Sparkes, I. A. 2009. A comparative study of the involvement of 17 Arabidopsis myosin family members on the motility of Golgi and other organelles. Plant Physiol. 150:700-709.

Blancaflor, E. B., Wang, Y. S., and Motes, C. M. 2006. Organization and function of the actin cytoskeleton in developing root cells. Int. Rev. Cytol. 252:219-264. 
Campellone, K. G., and Welch, M. D. 2010. A nucleator arms race: Cellular control of actin assembly. Nat. Rev. Mol. Cell Biol. 11:237-251.

Cárdenas, L., Vidali, L., Domnguez, J., Prez, H., Snchez, F., Hepler, P. K. and Quinto, C. 1998. Rearrangement of actin microfilaments in plant root hairs responding to Rhizobium etli nodulation signals. Plant Physiol. 116:871-877.

Davidson, A. L., and Newcomb, W. 2001. Changes in actin microfilament arrays in developing pea root nodule cells. Can. J. Bot. 79:767-776.

Day, B., Henty, J. L., Porter, K. J., and Staiger, C. J. 2011. The pathogenactin connection: A platform for defense signaling in plants. Annu. Rev. Phytopathol. 49:483-506.

de Ruijter, N. C. A., Bisseling, T., and Emons, A. M. C. 1999. Rhizobium Nod factors induce an increase in sub-apical fine bundles of actin filaments in Vicia sativa root hairs within minutes. Mol. Plant Microbe Interact. 12:829-832.

Deeks, M. J., and Hussey, P. J. 2005. Arp2/3 and SCAR: Plants move to the fore. Nat. Rev. Mol. Cell Biol. 6:954-964.

Deeks, M. J., Hussey, P. J., and Davies, B. 2002. Formins: Intermediates in signal-transduction cascades that affect cytoskeletal reorganization. Trends Plant Sci. 7:492-498.

Deeks, M. J., Calcutt, J. R., Ingle, E. K. S., Hawkins, T. J., Chapman, S., Richardson, A. C., Mentlak, D. A., Dixon, M. R., Cartwright, F., Smertenko, A. P., Oparka, K., and Hussey, P. J. 2012. A superfamily of actin-binding proteins at the actin-membrane nexus of higher plants. Curr. Biol. 22:1595-1600.

Dominguez, R. 2009. Actin filament nucleation and elongation factorsStructure-function relationships. Crit. Rev. Biochem. Mol. Biol. 44: 351-366.

Eitzen, G., Wang, L., Thorngren, N., and Wickner, W. 2002. Remodeling of organelle-bound actin is required for yeast vacuole fusion. J. Cell Biol. 158:669-679.

Fedorova, E. E., de Felipe, M. R., Pueyo, J. J., and Lucas, M. M. 2007. Conformation of cytoskeletal elements during the division of infected Lupinus albus L. nodule cells. J. Exp. Bot. 58:2225-2236.

Gavrin, A., Kaiser, B. N., Geiger, D., Tyerman, S. D., Wen, Z., Bisseling, T., and Fedorova, E. E. 2014. Adjustment of host cells for accommodation of symbiotic bacteria: Vacuole defunctionalization, HOPS suppression, and TIP1g retargeting in Medicago. Plant Cell 26:3809-3822.

Gopaldass, N., Patel, D., Kratzke, R., Dieckmann, R., Hausherr, S., Hagedorn, M., Monroy, R., Krüger, J., Neuhaus, E. M., Hoffmann, E., Hille, K., Kuznetsov, S. A., and Soldati, T. 2012. Dynamin A, Myosin IB and Abp1 couple phagosome maturation to F-actin binding. Traffic 13: 120-130.

Hawkins, T. J., Deeks, M. J., Wang, P., and Hussey, P. J. 2014. The evolution of the actin binding NET superfamily. Front. Plant Sci. 5:254.

Henty-Ridilla, J. L., Shimono, M., Li, J., Chang, J. H., Day, B., and Staiger, C. J. 2013. The plant actin cytoskeleton responds to signals from microbe-associated molecular patterns. PLoS Pathog. 9:e1003290.

Hossain, M. S., Liao, J., James, E. K., Sato, S., Tabata, S., Jurkiewicz, A., Madsen, L. H., Stougaard, J., Ross, L., and Szczyglowski, K. 2012. Lotus japonicus ARPC1 is required for rhizobial infection. Plant Physiol. 160 917-928.

Ivanov, S., Fedorova, E. E., Limpens, E., De Mita, S., Genre, A., Bonfante, P., and Bisseling, T. 2012. Rhizobium-legume symbiosis shares an exocytotic pathway required for arbuscule formation. Proc. Natl. Acad. Sci. U.S.A. 109:8316-8321.

Kereszt, A., Mergaert, P., and Kondorosi, E. 2011. Bacteroid development in legume nodules: Evolution of mutual benefit or of sacrificial victims? Mol. Plant Microbe Interact. 24:1300-1309.

Ketelaar, van der Honin H.S., and Emons, A.M.C. 2010. Probing cytoplasmic organization and the actin cytoskeleton of plant cells with optical tweezers. Biochem. Soc. Trans. 38:823-828.

Kotchoni, S. O., Zakharova, T., Mallery, E. L., Le, J., El-Assal, Sel.-D., and Szymanski, D. B. 2009. The association of the Arabidopsis actin-related protein $2 / 3$ complex with cell membranes is linked to its assembly status but not its activation. Plant Physiol. 151:2095-2109.

Kumar, Y., and Valdivia, R. H. 2008. Actin and intermediate filaments stabilize the Chlamydia trachomatis vacuole by forming dynamic structural scaffolds. Cell Host Microbe 4:159-169.

Liebl, D., and Griffiths, G. 2009. Transient assembly of F-actin by phagosomes delays phagosome fusion with lysosomes in cargooverloaded macrophages. J. Cell Sci. 122, Pt 16:2935-2945.

Limpens, E., Ramos, J., Franken, C., Raz, V., Compaan, B., Franssen, H., Bisseling, T., and Geurts, R. 2004. RNA interference in Agrobacterium rhizogenes-transformed roots of Arabidopsis and Medicago truncatula. J. Exp. Bot. 55:983-992.

Limpens, E., Ivanov, S., van Esse, W., Voets, G., Fedorova, E., and Bisseling, T. 2009. Medicago N2-fixing symbiosomes acquire the endocytic identity marker Rab7 but delay the acquisition of vacuolar identity. Plant Cell 21:2811-2828.

Limpens, E., Moling, S., Hooiveld, G., Pereira, P. A., Bisseling, T., Becker, J. D., and Küster, H. 2013. Cell- and tissue-specific transcriptome analyses of Medicago truncatula root nodules. PLoS ONE 8:e64377.

Marchetti, M., Capela, D., Poincloux, R., Benmeradi, N., Auriac, M.-C., Le $\mathrm{Ru}$, A., Maridonneau-Parini, I., Batut, J., and Masson-Boivin, C. 2013 Queuosine biosynthesis is required for Sinorhizobium meliloti-induced cytoskeletal modifications on HeLa cells and symbiosis with Medicago truncatula. PLoS ONE 8:e56043.

Miyahara, A., Richens, J., Starker, C., Morieri, G., Smith, L., Long, S., Downie, J. A., and Oldroyd, G. E. D. 2010. Conservation in function of a SCAR/WAVE component during infection thread and root hair growth in Medicago truncatula. Mol. Plant Microbe Interact. 23:1553-1562.

Morel, E., Parton, R. G., and Gruenberg, J. 2009. Annexin A2-dependent polymerization of actin mediates endosome biogenesis. Dev. Cell 16: 445-457.

Pollard, T. D. 2007. Regulation of actin filament assembly by Arp2/3 complex and formins. Annu. Rev. Biophys. Biomol. Struct. 36:451-477

Pollard, T. D., and Cooper, J. A. 2009. Actin, a central player in cell shape and movement. Science 326:1208-1212.

Rotty, J. D., Wu, C., and Bear, J. E. 2013. New insights into the regulation and cellular functions of the ARP2/3 complex. Nat. Rev. Mol. Cell Biol. 14:7-12.

Roux, B., Rodde, N., Jardinaud, M.-F., Timmers, T., Sauviac, L., Cottret, L., Carrère, S., Sallet, E., Courcelle, E., Moreau, S., Debellé, F., Capela, D. de Carvalho-Niebel, F., Gouzy, J., Bruand, C., and Gamas, P. 2014. An integrated analysis of plant and bacterial gene expression in symbiotic root nodules using laser-capture microdissection coupled to RNA sequencing. Plant J. 77:817-837.

Sinclair, A., Schenkel, M., and Mathur, J. 2009. Signaling to the actin cytoskeleton during cell morphogenesis and patterning. Pages 135-153 in: Signaling in Plants. F. Baluska and S. Mancuso, Springer, Heidelberg, Germany.

Takemoto, D., and Hardham, A. R. 2004. The cytoskeleton as a regulator and target of biotic interactions in plants. Plant Physiol. 136:3864-3876.

Taunton, J., Rowning, B. A., Coughlin, M. L., Wu, M., Moon, R. T., Mitchison, T. J., and Larabell, C. A. 2000. Actin-dependent propulsion of endosomes and lysosomes by recruitment of N-WASP. J. Cell Biol. 148:519-530.

Timmers, A. C. J. 2008. The role of the plant cytoskeleton in the interaction between legumes and rhizobia. J. Microsc. 231:247-256.

Untergasser, A., Nijveen, H., Rao, X., Bisseling, T., Geurts, R., and Leunissen, J. A. M. 2007. Primer3Plus, an enhanced web interface to Primer3. Nucleic Acids Res. 35:W71-W74.

Van de Velde, W., Zehirov, G., Szatmari, A., Debreczeny, M., Ishihara, H., Kevei, Z., Farkas, A., Mikulass, K., Nagy, A., Tiricz, H., Satiat-Jeunemaître, B., Alunni, B., Bourge, M., Kucho, K., Abe, M., Kereszt, A., Maroti, G., Uchiumi, T., Kondorosi, E., and Mergaert, P. 2010. Plant peptides govern terminal differentiation of bacteria in symbiosis. Science 327:1122-1126.

van der Honing, H. S., Emons, A. M. C., and Ketelaar, T. 2007. Actin based processes that could determine the cytoplasmic architecture of plant cells. BBA Mol. Cell Res. 1773:604-614.

Van Gestel, K., Slegers, H., Von Witsch, M., Šamaj, J., Baluška, F., and Verbelen, J. P. 2003. Immunological evidence for the presence of plant homologues of the actin- related protein Arp3 in tobacco and maize: Subcellular localization to actin-enriched pit fields and emerging root hairs. Protoplasma 222:45-52.

Vasse, J., de Billy, F., Camut, S., and Truchet, G. 1990. Correlation between ultrastructural differentiation of bacteroids and nitrogen fixation in alfalfa nodules. J. Bacteriol. 172:4295-4306.

Wang, D., Griffitts, J., Starker, C., Fedorova, E., Limpens, E., Ivanov, S., Bisseling, T., and Long, S. 2010. A nodule-specific protein secretory pathway required for nitrogen-fixing symbiosis. Science 327:1126-1129.

Welch, M. D., and Way, M. 2013. Arp2/3-mediated actin-based motility: A tail of pathogen abuse. Cell Host Microbe 14:242-255.

Whitehead, L. F., and Day, D. A. 1997. The peribacteroid membrane. Physiol. Plant. 100:30-44.

Whitehead, L., Day, D., and Hardham, A. 1998. Cytoskeletal arrays in the cells of soybean root nodules: The role of actin microfilaments in the organisation of symbiosomes. Protoplasma 203:194-205.

Yanagisawa, M., Zhang, C., and Szymanski, D. B. 2013. ARP2/3dependent growth in the plant kingdom: SCARs for life. Front. Plant Sci. 4:166.

Yokota, K., Fukai, E., Madsen, L. H., Jurkiewicz, A., Rueda, P., Radutoiu, S., Held, M., Hossain, M. S., Szczyglowski, K., Morieri, G., Oldroyd G. E. D., Downie, J. A., Nielsen, M. W., Rusek, A. M., Sato, S., Tabata S., James, E. K., Oyaizu, H., Sandal, N., and Stougaard, J. 2009. 
Rearrangement of actin cytoskeleton mediates invasion of Lotus japonicus roots by Mesorhizobium loti. Plant Cell 21:267-284.

Zepeda, I., Sánchez-López, R., Kunkel, J. G., Bañuelos, L. A., HernándezBarrera, A., Sánchez, F., Quinto, C., and Cárdenas, L. 2014 Visualization of highly dynamic F-actin plus ends in growing phaseolus vulgaris root hair cells and their responses to Rhizobium etli nod factors. Plant Cell Physiol. 55:580-592.

Zhang, X., Dyachok, J., Krishnakumar, S., Smith, L. G., and Oppenheimer, D. G. 2005. IRREGULAR TRICHOME BRANCH1 in Arabidopsis encodes a plant homolog of the actin-related protein $2 / 3$ complex activator Scar/WAVE that regulates actin and microtubule organization Plant Cell 17:2314-2326.

Zhang, C., Mallery, E. L., and Szymanski, D. B. 2013. ARP2/3 localization in Arabidopsis leaf pavement cells: A diversity of intracellular pools and cytoskeletal interactions. Front. Plant Sci. 4:238.

\section{AUTHOR-RECOMMENDED INTERNET RESOURCE}

INRA Symbimix database: iant.toulouse.inra.fr/symbimics 